\title{
Nonholonomic System Modelling and Control with Single Degree of Freedom
}

\author{
Emmanuel Kwame Mensah \\ Department of Mathematics, Faculty of Engineering, Ghana Technology University College, PMB 100, Accra- \\ North, Ghana
}

\begin{abstract}
This paper considers nonholonomic system modelling and control of a single degree of freedom. The model is based on a linear ordinary differential equation using the principles of vibrations in the area of feedback control system which is applied in many industrial applications. In this field, actual motion deviate significantly from the desired motion, and as a result of this deviation, performance, precision and accuracy of the system may not be acceptable. The problem is solved using the principles of PID and Routh-Hurwitz criterion of stability. At end the system was stable and the actual motion is the same as desired motion. The system was controllable and observable. Keywords: Nonholonomic system, PID, Routh-Hurwitz stability criterion, Controllability, Observability DOI: $10.7176 / \mathrm{CTI} / 10-04$
\end{abstract}

Publication date:July $31^{\text {st }} 2020$

\section{Introduction}

In applications of theories in solving of problems of motion and equilibrium of mechanical systems, one may make use of the constraints to unearth a hidden fact about the systems under the discussion. One in which the mechanical state of a given system is defined by a finite number of parameters that can completely describe the position of the system at any given time. In this description certain conditions arises which are handicap of the system. These handicaps conditions are termed constrains. In terms of this, systems are classified as holonomic and nonholonomic. Mathematically, Holonomic system are systems in which all constraints are integrable into positional constraints of the form $f\left(q_{1}, q_{2}, \ldots, q_{n}, t\right)=0 \forall q_{i} \in \mathfrak{R}^{n}$ and $t$ is time. In such a system, it can be used to reduce the number of degrees of freedom in the system. In case of the nonholonomic systems, it cannot be used to reduce the number of degrees of freedom in the system and can be defined as systems which have constraints that are nonintegrable into positional constraints. Intuitively, Holonomic system where a robot can move in any direction in the configuration space whereas Nonholonomic systems are systems where the velocities (magnitude and or direction) and other derivatives of the position are constraint and can not move in any direction in the configuration space. Moreover, the controllable degree of freedom is less than the total degrees of freedom, then it is known as non-Holonomic drive. A car has three degrees of freedom; i.e. its position in two axes and its orientation. However, there are only two controllable degrees of freedom which are acceleration (or braking) and turning angle of steering wheel. This makes it difficult for the driver to turn the car in any direction (unless the car skids or slides). Hence a car is nonholonomic system.

The origin of nonholonomic system can be divided into two classes namely:

- Bodies of motion in contact with each other as they roll/move without slippage.

- Conservation of moments in a multi-body system associated with under-actuated control (Neimark \& Fufaev, 1972).

For the Unicycle example nonholonomy arises because at the touching point between disk and surface, the velocity are confined to be aligned with the heading angle, no slippage is allowed. For UAV model, since the engine thrust is always aligned with body's longitudinal direction, it can be considered approximately that there is no side slippage. For the car-like model the two nonholonomic constraints arise because there are no side slippage at both front and rear wheel. For the hopping robot model nonholonomy arises because when it flies in the air, the angular moment is conserved since there is no external force applied to the system . (Neimark \& Fufaev, 1972).

This paper we shall deal with nonholonomic systems with a single degree of freedom and the focus was robot. An important application of the theory of vibration is the area of feedback control system. In many industrial applications, a robot system is designed to perform, with high precision of a specified task or follow a desired motion. However, due to disturbances or the effect of unknown parameters such as friction, wear, clearances in the joint etc., the desired motion of the system cannot be achieved. The actual motion deviates significantly from the desired motion, and as a result of this deviation, performance, precision and accuracy of the system is not be acceptable. It is therefore important to be able to deal with this problem by proper design of a control system that automatically reduces this deviation and if possible, eliminates it.

\section{Concept of Ordinary Differential Equations in Relation to Vibration Mechanics}

In this section mathematical concept underpinning vibration mechanics will be considered. Below are the strands 
showing the flow of discussion:

- Second-order ordinary linear differential equation with constant coefficient

- Vibration Mechanics

\subsection{Second-Order Ordinary Linear Differential Equation with Constant Coefficient}

Application of Newton's second law to the study of motion of physical systems leads to second-order ordinary differential equations. Generally, in order to examine, understand, and analyse the behaviour of physical systems, we must first solve differential equations that governs vibrations of systems.

Considering $n t h-$ order ordinary linear differential equation with constant coefficient of the form

$$
a_{n} y^{(\mathrm{n})}+a_{n-1} y^{(n-1)}+\ldots .+a_{2} y^{(2)}+a_{1} y^{(1)}+a_{0} y=f(\mathrm{y})
$$

with the coefficient $a_{1}, \forall i=0,1,2, \ldots, n$ are real constants,$a_{n} \neq 0$ and if $f(\mathrm{y}) \neq 0$ the equation is known as nonhomogeneous .

For homogeneous equation $f(\mathrm{y})=0$ and we have

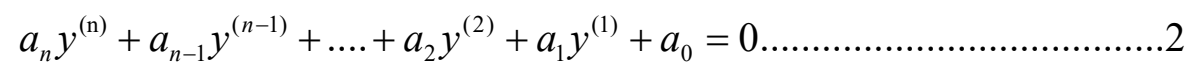

We begin by considering the special case where $n=2$ for homogeneous case. That is

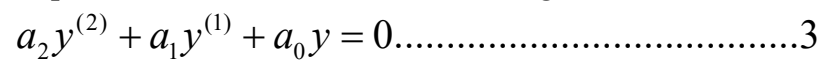

In terms of linear theory of vibration mechanics, $a_{0}, a_{1}$ and $a_{2}$ are constant coefficient that represent respectively stiffness, damping and inertia constants. In equation $1 f(x)$ is forcing function.

The auxiliary (characteristic) equation for equation 2.3 is given as

$$
a_{2} \eta^{2}+a_{1} \eta+a_{0}=0
$$

where $y=e^{\eta t} \neq 0$. The roots of quadratic equation in equation 2.4 is

$$
\eta_{i}=0.5 a_{2}^{-1}\left(-a_{1} \pm \sqrt{a_{1}^{2}-4 a_{2} a_{0}}\right) \quad \forall i=1,2
$$

There exist three forms of the general solution to equation 2.3 corresponding to the three cases:

Case I: $\eta_{1}$ and $\eta_{2}$ are real and distinct $\left(a_{1}^{2}-4 a_{2} a_{0}>0\right)$

Case II: $\eta_{1}$ and $\eta_{2}$ are real and equal $\left(a_{1}^{2}-4 a_{2} a_{0}=0\right)$

Case III: $\eta_{1}$ and $\eta_{2}$ are conjugate complex numbers $\left(a_{1}^{2}-4 a_{2} a_{0}<0\right)$

\section{Case I: Distinct Real Roots}

$$
y(t)=c_{1} e^{\eta_{1} t}+c_{2} e^{\eta_{2} t}
$$

Case II: Repeated Real Roots

$$
y(t)=c_{1} e^{\eta_{1} t}+c_{2} t e^{\eta_{1} t}
$$

\section{Case III: Conjugate Complex Roots}

In this particular case $\eta_{i}=\alpha \pm j \beta \quad \forall i=1,2$.

$$
y(t)=e^{\alpha t}\left(c_{1} \cos \beta t+c_{2} \sin \beta t\right)
$$

In all the equations from 6 to $8, c_{1}$ and $c_{2}$ are constants.

\subsection{Controllability and Observability of Systems}

Considering a system of the form

$$
\left.\begin{array}{l}
\& \&=A x(t)+B u(t) \\
y=C x(t)+D u(t)
\end{array}\right\}
$$

where $A, B, C$, and $D$ are respectively $n \times n, n \times 1,1 \times n, 1 \times n$ matrices.

The sufficiently condition for complete state controllability is that the $n \times n$ controllability matrix,

$$
M_{C}=\left[B: A B: A^{2} B: \ldots: A^{n-1} B\right]
$$

contain $n$ linearly independent row or column vectors (i.e. rank $n$ ) that is, the matrix is non-singular. In 
this wise the rank of the controllability matrix is equal to the order of the system.

Moreover, for complete observability, if $n \times n$ the observability matrix.

$$
N_{O B}=\left[C^{T}: A^{T} C^{T}: \ldots:\left(A^{T}\right)^{n-1} C^{T}\right]
$$

is of rank $n$,i.e. is non-singular having a non-zero determinant.

\subsection{Vibration Mechanics}

The coefficient of second order ordinary differential equation in application to mechanics are acceleration, velocity and displacement. As already mentioned in the previous section, the coefficients also represent physical parameters such as inertia, damping, and restoring elastic forces. These coefficients not only have a significant effect on the response of the mechanical and structural system, but they also affect the stability as well as the speed response of the system to a given excitation. Changes in these coefficients may result in a stable and unstable system, and /or oscillatory or non- oscillatory system. In all this situations, control of vibration is critical and important (Inman, 2017)

\section{Mathematical model of Robotic Arm}

In order to demonstrate the use of control system theory based on mathematical principles in solving the problem stated, we consider industrial single robotic arm as illustrated figure 1 below.

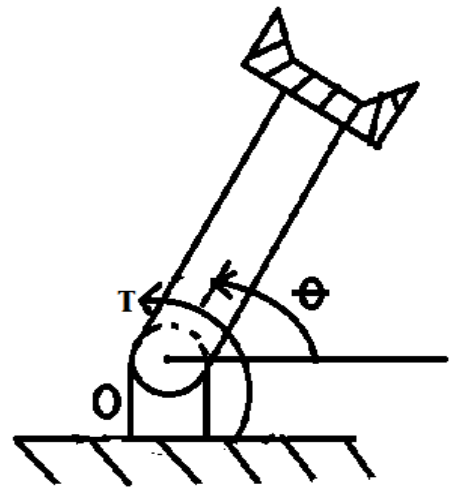

Figure 1. Motion control of a single robotic arm

Suppose $T$ is the torque applied by motor placed at the joint at $O$.

The equation of motion of the system is given in terms of angular orientation of the arm as

$$
I_{0} \theta^{(2)}+\frac{l}{2} m g \cos (\theta)=T
$$

Where $I_{0}$ is the mass of the inertia of the robotic arm defined with respect to point $O, m$ and $l$ are, respectively, mass and length of the arm. Moreover, $g$ is the gravitational constant, and $\theta$ is the angular orientation of the arm as shown in figure 1. For any applied torque, equation 1 represent the motion of the robotic arm and if $\theta_{a}$ denotes actual motion, equation 1 can be written as

$$
I_{0} \theta_{a}^{(2)}+\frac{l}{2} m g \cos \left(\theta_{a}\right)=T
$$

Due to disturbances and the effect of motion unknown parameters whose effect is not account for in equation 2 , the actual motion $\theta_{a}$ may deviates from the desired motion $\theta_{d}$. In order to obtain desired motion $\theta_{d}$, we choose the motor torque $T$ in the form

$$
I_{0} \theta_{d}^{(2)}-k_{v}\left(\theta_{a}^{(1)}-\theta_{d}^{(1)}\right)-k_{p}\left(\theta_{a}-\theta_{d}\right)+\frac{l}{2} m g \cos \left(\theta_{a}\right)=T \text {. }
$$

where $k_{v}$ and $k_{p}$ represent velocity and position gain respectively which is selected in such a manner that the deviation from the desired displacement is eliminated.

\section{Assumptions}

i. $\quad \theta_{d}, \theta_{d}^{(1)}$ and $\theta_{d}^{(2)}$ are assumed to be known because of the desired motion is assumed to be specific.

ii. The inertia properties and dimension of the robotic arm are assumed to be known. 
Using proper sensors to measure the actual displacement $\theta_{a}$ and its derivatives due to the fact that the sensor measure the angular orientation, velocity and acceleration of the robotic arm during the actual motion. At this point, equation 3 can be calculated and proper signal is given to motion in order to produce this torque. Equating equations 2 and 3 , we have,

$I_{0}\left(\theta_{a}^{(2)}-\theta_{d}^{(2)}\right)-k_{v}\left(\theta_{a}^{(1)}-\theta_{d}^{(1)}\right)-k_{p}\left(\theta_{a}-\theta_{d}\right)=0$

Since the error is the deviation of the actual motion from the desired motion, we have

$$
\left.\begin{array}{l}
e(t)=\theta_{d}(t)-\theta_{a}(t) \\
e^{(1)}(t)=\theta_{d}^{(1)}(t)-\theta_{a}^{(1)}(t) \\
e^{(2)}(t)=\theta_{d}^{(2)}(t)-\theta_{a}^{(2)}(t)
\end{array}\right\}
$$

Substitute equation 3.5 into equation 3.4 , we have

$$
I_{0} e^{(2)}(t)+k_{v} e^{(1)}+k_{p} e=0
$$

which is damped single degree of freedom system. The velocity and position gain ( i.e. $k_{v}$ and $k_{p}$ ) is selected such that error which is the displacement of the actual motion from desired motion goes to zero faster as time elapses.

The natural frequency of oscillation $\omega$ of the error in equation 6 is given by

$$
\omega_{n}=\sqrt{k_{p} I_{0}^{-1}}
$$

Damping coefficient, $C_{\text {damp }}$ is given by

$$
C_{\text {damp }}=2 I_{0} \omega_{n}=2 \sqrt{k_{p} I_{0}}
$$

The damping factor, $\zeta$ is found using the relation

$$
\zeta=\frac{k_{v}}{2 I_{0} \omega_{n}}=0.5 k_{v}\left(k_{p} I_{0}\right)^{-0.5}=\frac{k_{v}}{C_{\text {damp }}} .
$$

If the system is critically damped, $\zeta=1$. In this wise,

$$
\frac{k_{v}^{2}}{k_{p} I_{0}}=4
$$

For the error to approach zero in a relatively short time $k_{v}$ and $k_{p}$ should be selected such that it satisfies equation 21 (Inman, 2017) (Singiresu , 2018) .

\subsection{Control Systems of the Robotic Arm}

Figure 2 below illustrate the block diagram of the equation 3,

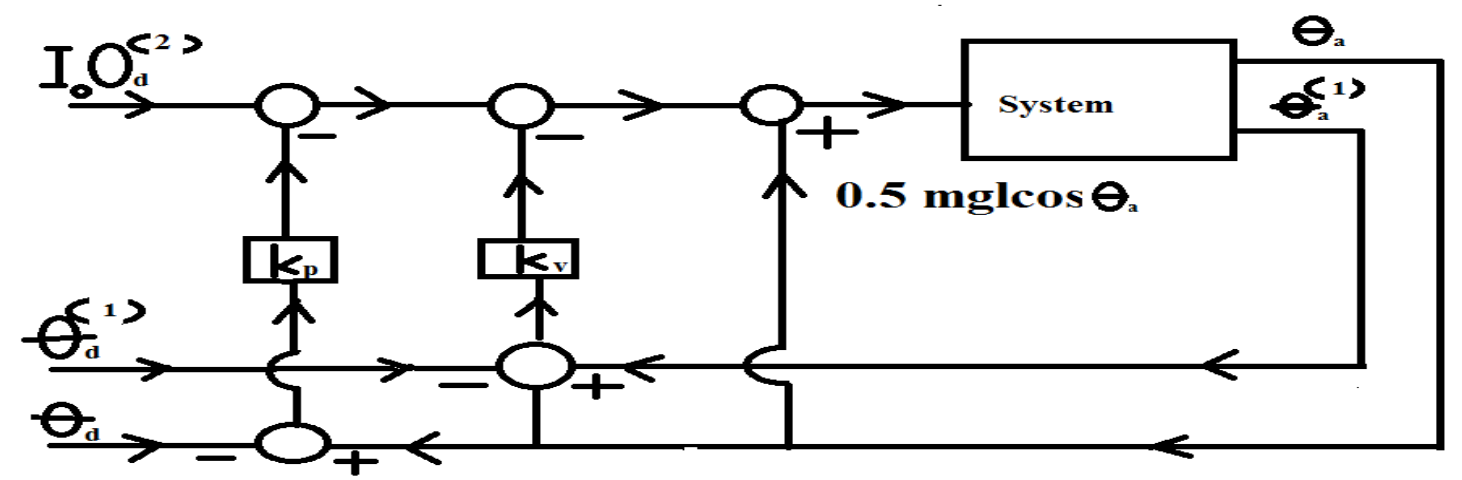

Figure 2. Block diagram

In this section, control action concept which is based on control law was used to further analyse the system. Considering equation 18, it is clear the control action is proportional -derivative (PD). Despite PD control action improves the transient response of the system, very effective when all the system parameter are known. Moreover, when there are no disturbances. Since some of the system parameters are not known in addition to existence of disturbances, the PD control will result in non-zero state error. Hence not appropriate. For Proportional - integral 
(PI) control action improves the steady state of the system. Due to this reason the combination of the two i.e. proportional -integral - derivative (PID) control action improves overall time response of the robotic arm system and also, solves all the problems the other two control actions could not perform. Hence PID was used in this situation. To be able to used it, an additional term $k_{i} \varepsilon$ was added to the torque $T$ in equation 18 given as

$$
I_{0} e^{(2)}(t)+k_{v} e^{(1)}+k_{p} e+k_{i} \varepsilon=0
$$

where $\varepsilon$ is defined as

$$
\varepsilon^{(1)}=e .
$$

Equation 23 and 24 must be solved simultaneously in order to determine the error as a function of time. From equation 24,

$$
\varepsilon=\int e d t
$$

Substitute equation 25 into equation 23 we have,

$$
I_{0} e^{(2)}(t)+k_{v} e^{(1)}(t)+k_{p} e(t)+k_{i} \int e(t) d t=0 .
$$

Equation 26 depends on equation 9 for determination of $k_{v}$ and $k_{p}$. Apart from this, the system should obey stability condition that is roots of 26 should negative or lie on the left part of the complex plane. This testify the motion stability of the of the system. Hence the error function should belong a family of decay functions according to the form of equations 6,7, and 8 (Inman, 2017) (Singiresu , 2018).

\section{Data Analysis}

The researcher used a of mass $7.2 \mathrm{~kg}$ and length of the $\operatorname{rod}(l)$ is $10 \mathrm{~m}$. Hence mass of inertia is given as

$$
I_{0}=\frac{m l^{2}}{12}=\frac{7.2(10)^{2}}{12}=60 \mathrm{kgm}^{2}
$$

From equation 9, we have

$$
\begin{aligned}
& \zeta=\frac{0.5 k_{v}}{\left(k_{p} I_{0}\right)^{0.5}} \\
& \frac{k_{v}^{2}}{k_{p} I_{0}}=4 \zeta^{2} \\
& k_{v}=\sqrt{4 \zeta^{2} k_{p} I_{0}}
\end{aligned}
$$

The researcher considered, $k_{p}=1$ and $I_{0}=60 \mathrm{kgm}^{2}$

$$
k_{v}=\sqrt{240 \zeta^{2}}
$$

Since the theory of robot depends largely on the principles of pendulum with the primary objective of oscillating, the researcher considered the condition of underdamping. For this condition the damping factor $\zeta<1$.

Now considering $\zeta=\sqrt{0.5}<1$,

$$
k_{v}=\sqrt{120}=10.95
$$

Equation 6 is rewritten as

$$
\left.60 e^{(2)}(t)+10.95 e^{(1)}(t)+e(t)=T \quad \forall T \in\right] 0, \infty[
$$

The simulation block diagram for equation 28 is given below. 


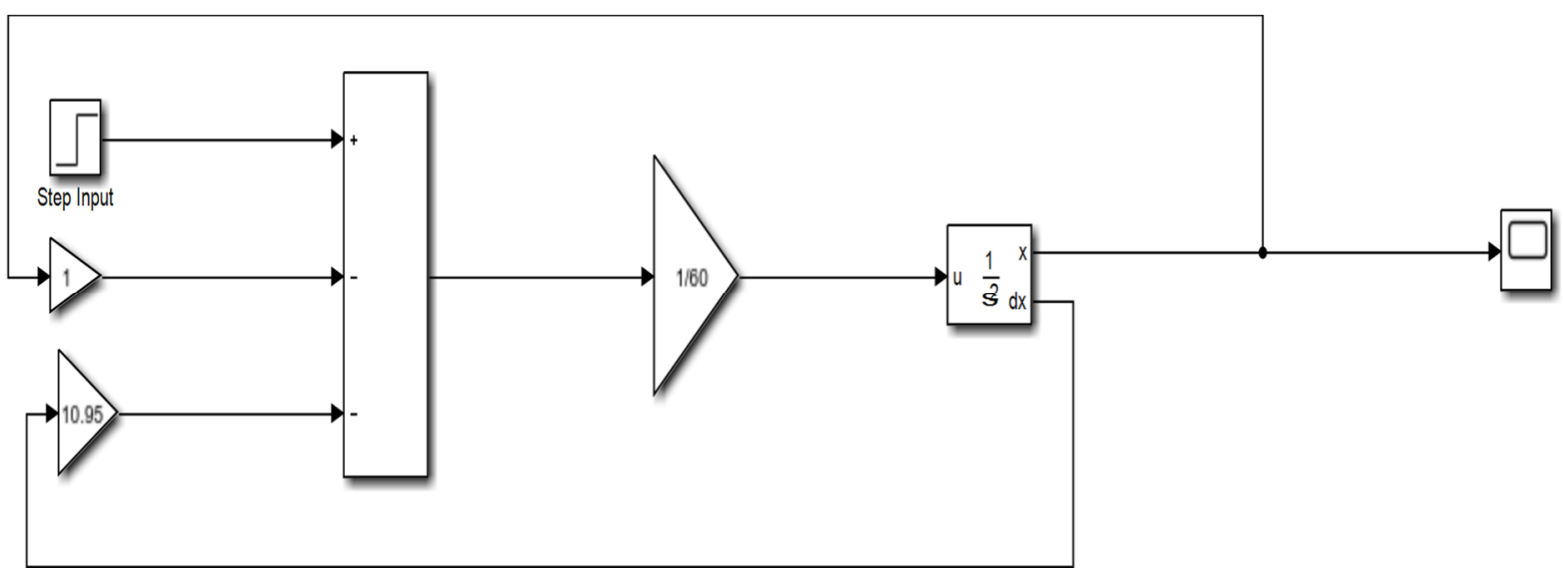

Figure 3: Simulation closed loop block diagram without integral controller

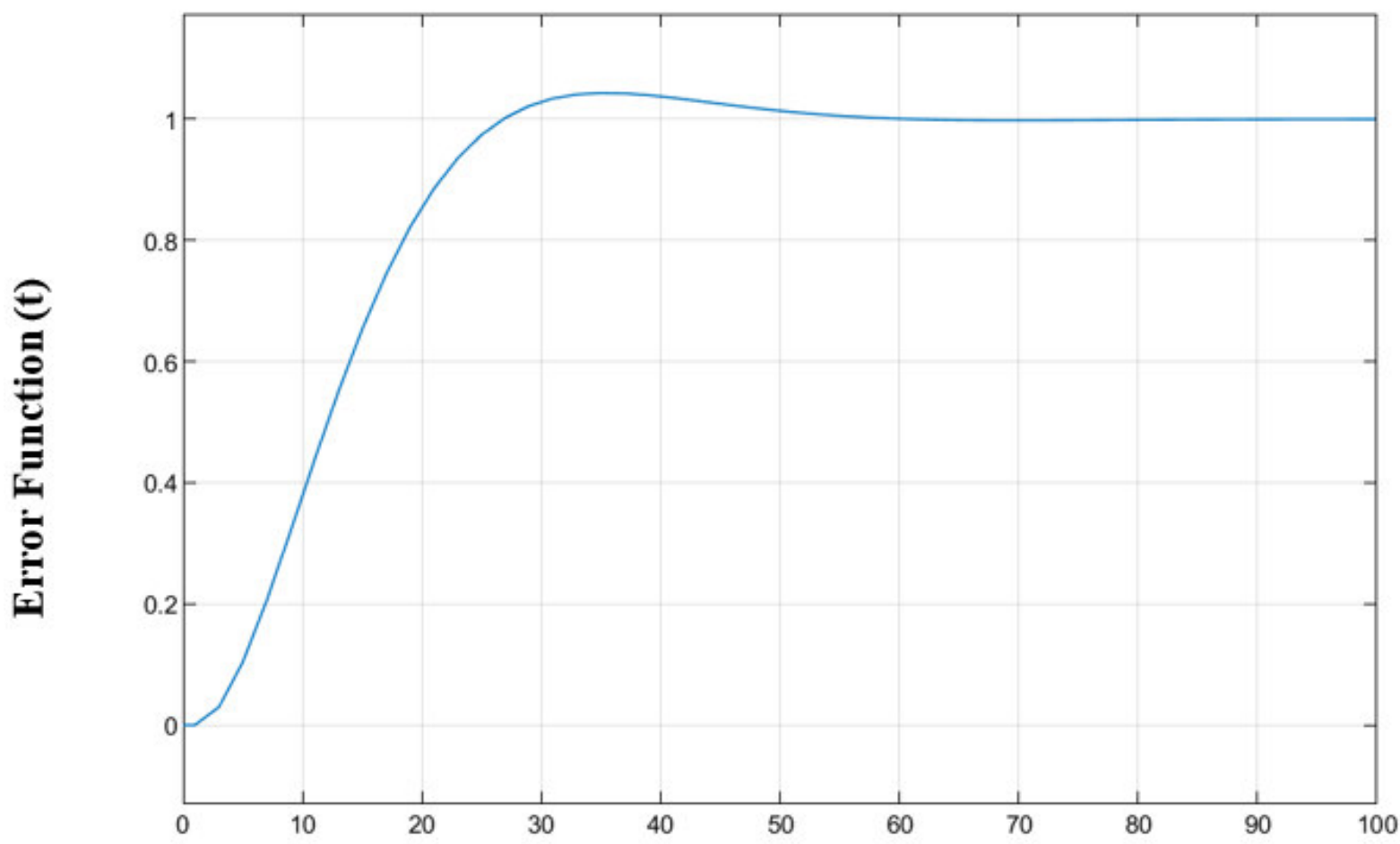

Offset $=0$

\section{Time (t)}

Figure 4. A graph of error function against time without Integral controller

Now From the graph in figure 4, it is clear the control action is proportional -derivative (PD). Despite PD control action improves the transient response of the system very effective when all the system parameter are known and when there are no disturbances. Since some of the system parameters are not known in addition to existence of disturbances, the PD control will result in non-zero state error. Hence not appropriate. For Proportional - integral (PI) control action improves the steady state of the system. Due to this reason the combination of the two i.e. proportional -integral - derivative (PID) control action improves overall time response of the robotic arm system. Hence PID was used in this situation. To be able to used it, an additional term $k_{i} \varepsilon$ was added to the torque $T$ in equation 6 . Therefor using equation 26 and substitution

$I_{0}=60 \mathrm{kgm}^{2}, k_{v}=10.95, k_{p}=1$ we have

$$
\left.60 e^{(2)}(t)+10.95 e^{(1)}(t)+e(t)+k_{i} \int e(t) d t=T \quad \forall T \in\right] 0, \infty[
$$

This equation 29 is an integral equation because the variable of interest is under the integral sign. Finding Laplace transform we have 


$$
\begin{aligned}
& E(s)\left(60 s^{2}+10.95 s+s+\frac{k_{i}}{s}\right)=T(s) \\
& \frac{E(s)}{T(s)}=\frac{s}{60 s^{3}+10.95 s^{2}+s+k_{i}}
\end{aligned}
$$

The choice of $k_{i}$ is critical in the determination of the stability of the system and marking error function of the system approaches zero as time elapses. Hence for stability, we solve the equation $60 s^{3}+10.95 s^{2}+s+k_{i}=0$ to determine the position of the poles in the complex plane. Using Routh -Hurwitz stability criterion we have,

$$
\begin{array}{cccc}
s^{3} & 60 & 1 & 0 \\
s^{2} & 10.95 & k_{i} & \\
& \frac{10.95-60 k_{i}}{1} & 0 & \\
s^{1} & \frac{10.95}{s^{0}} & k_{i} &
\end{array}
$$

For stability,

i. $\quad k_{i}>0$

ii. $\quad \frac{10.95-60 k_{i}}{10.95}>0$

Solving the inequality equations

$$
\left.0<k_{i}<0.1828 \Rightarrow k_{i} \in\right] 0,0.1825[
$$

This implies that any value of which lies within the interval of 0 to 0.1825 , the system will make the sable.

Now choosing $\left.k_{i}=0.02 \in\right] 0,0.1825[$, we have

Solving this cubic equation, we have

$$
60 s^{3}+10.95 s^{2}+s+0.02=0
$$

$$
s=-0.02663, \quad-0.7793 \pm 0.08026 j
$$

Since the Real parts of the poles are located in the negative part of the complex plane, the system is stable under Routh -Hurwitz criterion. This also implies that

$$
\begin{aligned}
& \frac{E(s)}{T(s)}=\frac{(s-0)}{(s+0.02663)(s+0.7793+0.08026 j)(s+0.7793+0.08026 j)} \\
& E(s)=\frac{(s-0)}{(s+0.02663)(s+0.7793+0.08026 j)(s+0.7793+0.08026 j)} T(s)
\end{aligned}
$$

Considering $T(s)=U(s)=\frac{1}{s}$ we have the simulation block diagram,

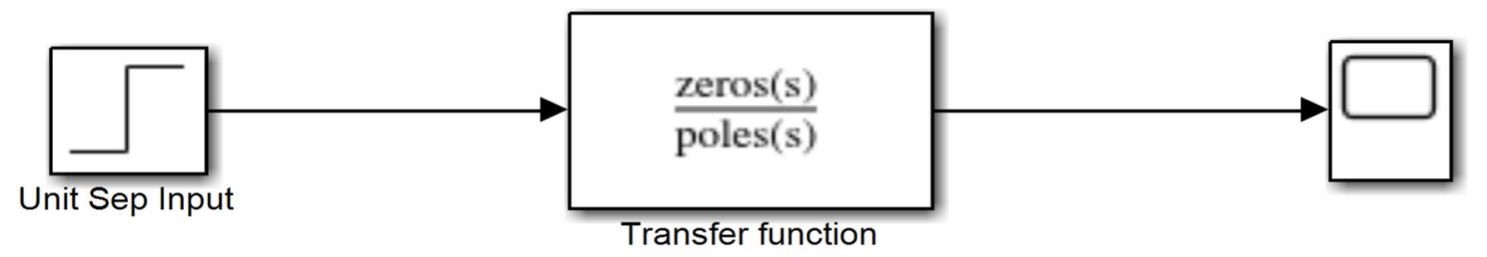

Figure 5. Simulation Open loop- Block diagram with Integral Controller 


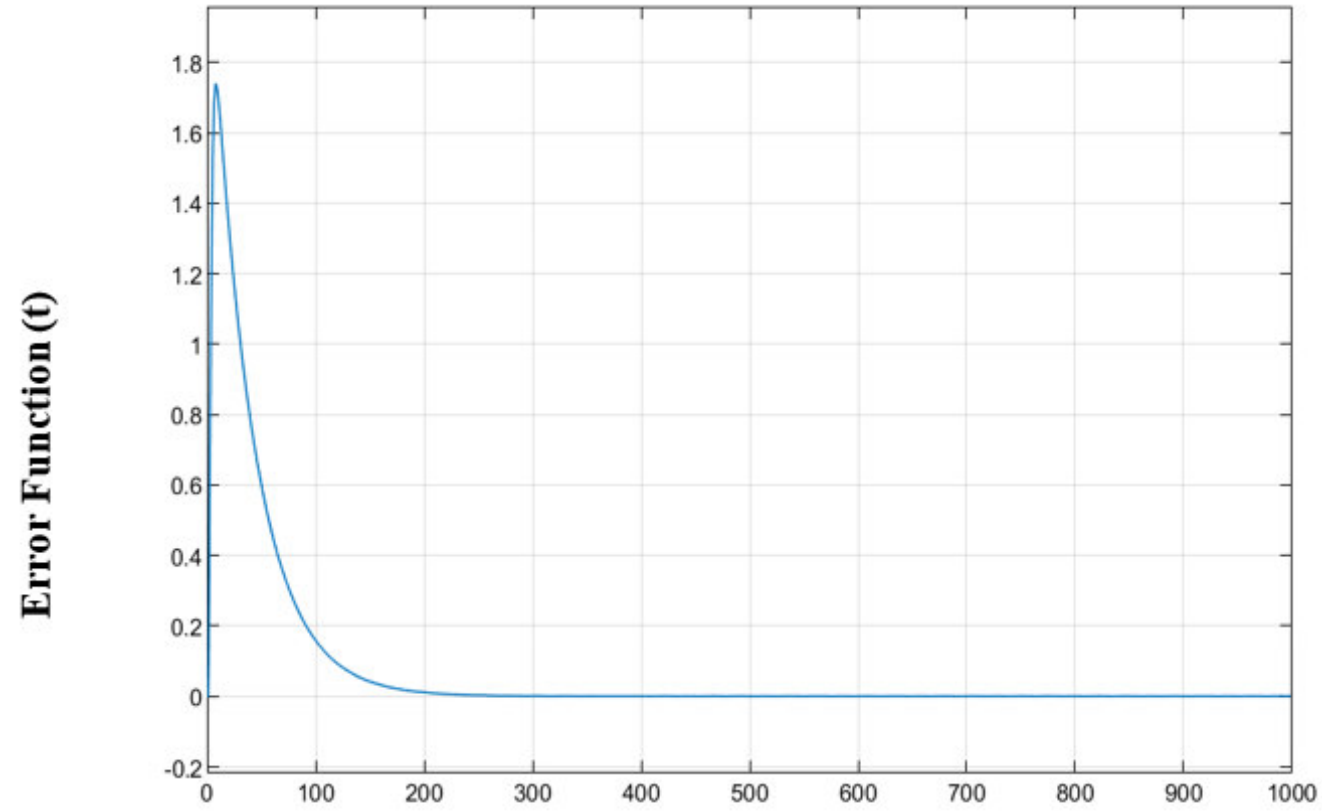

\section{Time (t)}

Figure 6. A graph of error function against Time with Integral controller

From the graph in figure 6, it is clear that the errors in the system has been eliminated as time elapses which means that actual motion is the same as desired motion. The graph also indicates the system is stable. Moreover, reliability of a system is a function of errors in the system. Since the error function moving to zero as time elapses implies that the system is reliable. Furthermore, it also implies the system is controllable and observable. The researcher moves further to verify whether the system is totally controllable and observable or not.

\subsection{Controllability of the System}

Converting equation 29 into state space form where we have

$$
\begin{aligned}
& y_{1}=\int e(t) d t \\
& y_{2}=y \\
& y_{3}=1 \& \\
& \psi_{3}=-0.02 y_{1}-y_{2}-10.95 y_{3}+T \\
& \& \&=\left(\begin{array}{l}
\& \& \\
\& \\
\& \\
\&
\end{array}\right)=\left(\begin{array}{ccc}
0 & 1 & 0 \\
0 & 0 & 1 \\
-0.02 & -1 & -10.95
\end{array}\right)\left(\begin{array}{l}
y_{1} \\
y_{2} \\
y_{3}
\end{array}\right)+\left(\begin{array}{l}
0 \\
0 \\
1
\end{array}\right) T \\
& \int e(t) d t=\left[\begin{array}{lll}
1 & 0 & 0
\end{array}\right]\left(\begin{array}{l}
y_{1} \\
y_{2} \\
y_{3}
\end{array}\right)
\end{aligned}
$$

From equations 10 and 11 ,

$$
\left.A=\left(\begin{array}{ccc}
0 & 1 & 0 \\
0 & 0 & 1 \\
-0.02 & -1 & -10.95
\end{array}\right), \quad B=\left(\begin{array}{l}
0 \\
0 \\
1
\end{array}\right), \quad C=\left[\begin{array}{lll}
1 & 0 & 0
\end{array}\right], \quad D=0\right\}
$$




$$
\begin{gathered}
A B=\left(\begin{array}{ccc}
0 & 1 & 0 \\
0 & 0 & 1 \\
-0.02 & -1 & -10.95
\end{array}\right)\left(\begin{array}{l}
0 \\
0 \\
1
\end{array}\right)=\left(\begin{array}{c}
0 \\
1 \\
-10.95
\end{array}\right) \\
A^{2} B=A(A B)=\left(\begin{array}{ccc}
0 & 1 & 0 \\
0 & 0 & 1 \\
-0.02 & -1 & -10.95
\end{array}\right)\left(\begin{array}{c}
0 \\
1 \\
-10.95
\end{array}\right)=\left(\begin{array}{c}
1 \\
-10.95 \\
118.9025
\end{array}\right) \\
\text { Controllability matrix, } M_{c}=\left[\begin{array}{lll}
B & A B & A^{2} B
\end{array}\right]=\left[\begin{array}{ccc}
0 & 0 & 1 \\
0 & 1 & -10.95 \\
1 & -10.95 & 118.9025
\end{array}\right] \\
\left|M_{c}\right|=-1
\end{gathered}
$$

Since the determinant is non-zero, the rank is equal to the order of the system. Therefore, the system is state controllable.

\subsection{Observability of the System}

Using the values in equation 31

$$
C^{T}=\left(\begin{array}{l}
1 \\
0 \\
0
\end{array}\right) \text {, }
$$

\section{From equation 12,}

$$
\begin{aligned}
& A^{T} C^{T}=(C A)^{T}=\left(\left(\begin{array}{lll}
1 & 0 & 0
\end{array}\right)\left(\begin{array}{ccc}
0 & 1 & 0 \\
0 & 0 & 1 \\
-0.02 & -1 & -10.95
\end{array}\right)\right)^{T}=\left(\begin{array}{lll}
0 & 1 & 0
\end{array}\right)^{T}=\left(\begin{array}{l}
0 \\
1 \\
0
\end{array}\right) \\
& \left(A^{T}\right)^{2} C^{T}=\left(\begin{array}{ccc}
0 & 0 & -0.02 \\
1 & 0 & -1 \\
0 & 1 & -10.95
\end{array}\right) C^{T}=\left(\begin{array}{ccc}
0 & -0.02 & 0.219 \\
0 & -1 & 10.93 \\
1 & -10.95 & 118.9025
\end{array}\right)\left(\begin{array}{l}
1 \\
0 \\
0
\end{array}\right)=\left(\begin{array}{l}
0 \\
0 \\
1
\end{array}\right)
\end{aligned}
$$

Observability matrix, $N_{O B}=\left[\begin{array}{lll}C^{T} & A^{T} C^{T} & \left(A^{T}\right)^{2} C^{T}\end{array}\right]=\left[\begin{array}{ccc}1 & 0 & 0 \\ 0 & 1 & 0 \\ 0 & 0 & 1\end{array}\right]$

$$
\left|N_{O B}\right|=1
$$

Since the determinant is non-zero, the rank is equal to the order of the system. Therefore, the system is state observable.

\section{Conclusion}

We conclude that the mathematically modelled single arm robot system, the actual motion is the same as desired motion since error function approaches zero as time elapses. Hence, the system is reliable in terms of executing its designed task. Moreover, the system is stable, controllable and observable.

\section{Reference}

Inman D.J.(2017), "Vibrations with control $2^{\text {nd }}$ edition", John Wiley \& Sons, Ltd, Hoboken, NJ, USA.

Neimark Ju. I. \& Fufaev N. A. (1972), "Dynamics of Nonholonomic Systems”, American Mathematical Society, USA.

Singiresu S. Rao (2018) “Mechanical Vibrations 6 ${ }^{\text {th }}$ edition”, Pearson Educational limited, Harlow, UK. 